\title{
ESTRUTURA DO COMPONENTE HERBÁCEO NO CAMPO NÃO INUNDÁVEL DE UMA RESTINGA EM PERNAMBUCO (BRASIL)
}

\author{
HERBACEOUS COMPONENT STRUCTURE IN THE NON-FLOODED FIELD OF RESTINGA IN PERNAMBUCO \\ STATE (BRAZIL)
}

\section{ESTRUCTURA DE COMPONENTES HERBÁCEOS EN EL CAMPO NO INUNDADO DE UNA RESTINGA EM EL ESTADODE PERNAMBUCO (BRASIL)}

\section{Resumo}

Eduardo Bezerra de Almeida Jr. Luann Brendo da Silva Costa ${ }^{2}$ Murielle Andreo Olivo ${ }^{3}$ Elcida de Lima Araújo ${ }^{4}$ Carmen Sílvia Zickel

A escassez de dados sobre a estrutura do estrato herbáceo na fisionomia campo dificulta a compreensão do arranjo e dinâmica das comunidades, da diversidade e dos padrões de distribuição das espécies entre diferentes ambientes. Diante disso, o presente estudo objetivou caracterizar a estrutura do componente herbáceo da fisionomia campo em uma área de restinga. As coletas foram realizadas na Reserva Particular do Patrimônio Natural Nossa Senhora do Outeiro de Maracaípe, em Pernambuco. Para a amostragem da comunidade herbácea foi utilizado o método de parcelas, sendo consideradas integrantes do estrato herbáceo as plantas não lenhosas com caule verde que possuíam altura entre 0,15 e $1 \mathrm{~m}$. Foram amostradas 29 espécies, 22 gêneros e nove famílias, totalizando 1.062 indivíduos. As famílias com maior riqueza foram Poaceae, Cyperaceae, Fabaceae, Euphorbiaceae e Rubiaceae. As espécies de maior VI foram Paspalum maritimum Trin., Cuphea flava Spreng., Eleocharis geniculata (L.) Roem. \& Schult., Stigmaphyllon paralias A. Juss., Rhynchospora barbata (Vahl) Kunth e Stylosanthes viscosa (L.) Sw. O Índice de diversidade de Shannon (H') foi de 2,414 e o Índice de Equabilidade de Pielou (J') encontrado foi de o,717. Podese concluir que as espécies $P$. maritimum, $R$. barbata e $S$. paralias destacaram-se no arranjo estrutural da fisionomia campo e que a vegetação apresentou espécies com ampla distribuição no Brasil.

\footnotetext{
1 Departamento de Biologia, Centro de Ciências Biológicas e da Saúde, Universidade Federal do Maranhão. Autor para correspondência: ebaj25@yahoo.com.br. ORCID: http://orcid.org/oooo-ooo17517-4775

2 Programa de Pós-graduação em Biodiversidade e Conservação, Departamento de Biologia, Laboratório de Estudos Botânicos, Universidade Federal do Maranhão. ORCID: http://orcid.org/oooo-0o03-29732961

3 Bióloga. ORCID: http://orcid.org/oooo-0oo2-2703-2999

4 Área de Botânica, Departamento de Biologia, Universidade Federal Rural de Pernambuco. ORCID: http://orcid.org/oooo-ooo2-3379-3540

5 Área de Botânica, Departamento de Biologia, Universidade Federal Rural de Pernambuco. ORCID: http://orcid.org/ oooo-ooo2-1323-4717
} 
Palavras-chave: diversidade, riqueza, vegetação litorânea, Reserva Particular do Patrimônio Natural.

\begin{abstract}
The lack of data on the structure of the herbaceous stratum in the field physiognomy makes it difficult to understand the arrangement and dynamics of the communities, of species diversity and distribution patterns among different environments. Therefore, the present study aimed to characterize the structure of the herbaceous component of the field physiognomy in a restinga area. Samples were collected in the Private Natural Heritage Reserve "Nossa Senhora of Outeiro of Maracaípe", Pernambuco State. Herb community was sampled using the plots method, considered as plants of the herbaceous stratum non-woody species with green stem that had a height between 0.15 and $1 \mathrm{~m}$. We sampled 29 species, 22 genera and nine families, totaling 1,062 individuals. The families richest were Poaceae, Cyperaceae, Fabaceae, Euphorbiaceae and Rubiaceae. The highest IV species were Paspalum maritimum Trin., Cuphea flava Spreng., Eleocharis geniculata (L.) Roem. \& Schult., Stigmaphyllon paralias A. Juss., Rhynchospora barbata (Vahl) Kunth e Stylosanthes viscosa (L.) Sw. Shannon Diversity Index (H') was from 2,414 and the Pielou Equity Index (J') was from 0,717 . It can be concluded that $P$. maritimum, $R$. barbata and $S$. paralias species stood out in the structural arrangement of the field physiognomy and that the vegetation presented species with wide distribution in Brazil.
\end{abstract}

Keywords: diversity, wealth, coastal vegetation, Private Natural Heritage Reserve.

\title{
Resumen
}

La falta de datos sobre la estructura del estrato herbáceo en la fisonomía de campo dificulta la comprensión de la disposición y la dinámica de las comunidades, de diversidad de especies y patrones de distribución entre diferentes ambientes. Por lo tanto, el presente estudio tuvo como objetivo caracterizar la estructura del componente herbáceo de la fisonomía de campo en un área de restinga. Las colecciones se realizaron en la Reserva Privada del Patrimonio Natural Nossa Senhora do Outeiro de Maracaípe, en el estado de Pernambuco. Para el muestreo de la comunidad herbácea se utilizó el método de parcelas, las plantas no leñosas con tallo verde con una altura entre 0,15 y $1 \mathrm{~m}$. Se muestrearon 29 especies, 22 géneros y nueve familias, con un total de 1,062 individuos. Las familias con mayor riqueza eran Poaceae, Cyperaceae, Fabaceae, Euphorbiaceae y Rubiaceae. Las especies más altas de VI fueron Paspalum maritimum Trin., Cuphea flava Spreng., Eleocharis geniculata (L.) Roem. \& Schult., Stigmaphyllon paralias A. Juss., Rhynchospora barbata (Vahl) Kunth e Stylosanthes viscosa (L.) Sw. El índice de diversidad de Shannon (H') fue 2,414 y el índice de equidad de Pielou (J') fue o,717. Se puede concluir que la especie $P$. maritimum, $R$. barbata y $S$. paralias se destacó en la disposición estructural de la fisonomía de la campo y que la vegetación presentaba especies con amplia distribución en Brasil.

Palabras clave: diversidad, riqueza, vegetación costera, Reserva Privada del Patrimonio Natural. 


\section{INTRODUÇÃO}

Os estudos fitossociológicos sobre o estrato herbáceo carecem de mais pesquisas e análises, considerando a grande diversidade de sua flora. Quando comparado ao componente lenhoso essa carência de dados se torna ainda mais evidente (Araújo et al. 2005; Munhoz e Felfili 2008).

A fisionomia da vegetação reflete a disposição, o arranjo e as relações entre os indivíduos que constituem a comunidade vegetal (Martins e Batalha 2011). Tais características podem variar dentro da mesma formação vegetal, sobretudo se esta ocupar extensas áreas com heterogeneidade de condições ambientais (Almeida Jr. et al. 2009).

Entre tais formações, destaca-se a restinga, que ocupa cerca de $80 \%$ da costa brasileira e engloba um conjunto de comunidades vegetais fisionomicamente distintas. As restingas são formações pioneiras e possuem uma vegetação própria adaptada às condições salinas e arenosas com influência de marés denominada vegetação halófila-psamófila, as quais se estabelecem rente à praia, gerando um mosaico de comunidades vegetais associadas à geomorfologia local e as diferenças ambientais (Sugiyama 1998; Almeida Jr. et al. 2009; Santos-Filho et al. 2015); e conforme vai se afastando da linha do mar, sentido continente, a vegetação herbácea vai apresentando diferentes formas de vida.

Nas restingas do Nordeste brasileiro, por exemplo, o estrato herbáceo contribui com até 50\% das listas florísticas (Sacramento et al. 2007; Almeida e Zickel 2009; Cantarelli et al. 2012; Silva et al. 2016; Amorim et al. 2016), mostrando a importância das ervas na riqueza total da área. Além disso, as espécies herbáceas terrícolas têm maior taxa de extinção natural do que plantas de outros estratos, resultando na perda do patrimônio natural (Fuhro et al. 2005; Gilliam 2007).

A escassez de dados sobre a estrutura do estrato herbáceo, sobretudo na fisionomia campo, dificulta a compreensão do arranjo e dinâmica das comunidades, da diversidade e dos padrões de distribuição das espécies entre diferentes ambientes, impossibilitando maiores análises sobre esse estrato, seja em ambientes conservados ou antropizados (Oliveira et al. 2013; Amorim et al. 2016). E do ponto de vista estrutural, pouco se conhece sobre o arranjo desse estrato em áreas de restinga e como essas espécies se desenvolvem nas diferentes fisionomias desse ecossistema (Amorim et al. 2016).

No Nordeste, os estudos que analisaram o estrato herbáceo foram realizados por Dias e Menezes (2007) e Menezes et al. (2012), no litoral da Bahia; Amorim et al. (2016), Araújo et al. (2016) e Santos et al. (2019), no litoral do Maranhão. Nesse contexto, analisar a flora herbácea das áreas de restinga pode contribuir para compreender o arranjo e distribuição das espécies nesse ecossistema, possibilitando a criação de planos de manejo e de recuperação de áreas degradadas, ou ainda, documentar as espécies e formas de vida existentes para estudos futuros nesses ambientes. Portanto, o presente estudo teve como objetivo descrever a estrutura do 
componente herbáceo da fisionomia campo não inundável em uma área de restinga no litoral sul de Pernambuco.

\section{MATERIAL E MÉTODOS}

\section{Área de estudo}

As coletas foram realizadas em 2005 na Reserva Particular do Patrimônio Natural (RPPN) Nossa Senhora do Outeiro de Maracaípe, município de Ipojuca (o8 $31^{\prime} 48^{\prime \prime S}$ e $35^{\circ}$ o1'o5” $^{\prime} \mathrm{W}$ ), litoral Sul de Pernambuco (Figura 1). A área possui cerca de 130 ha, dos quais 76,2 ha constituem-se de vegetação de restinga (Almeida Jr. et al. 2009).

O clima local é classificado como As' (tropical chuvoso com verão seco), evidenciando menos de $60 \mathrm{~mm}$ de chuva no mês mais seco (Köppen 1948) e precipitação anual média de $2.400 \mathrm{~mm}$ (INMET 2017). O solo é do tipo arenoso, com teores de areia variando de $98 \%$ a $100 \%$, sendo classificado como Neossolo Quartzarênico (Solos Embrapa 2013).

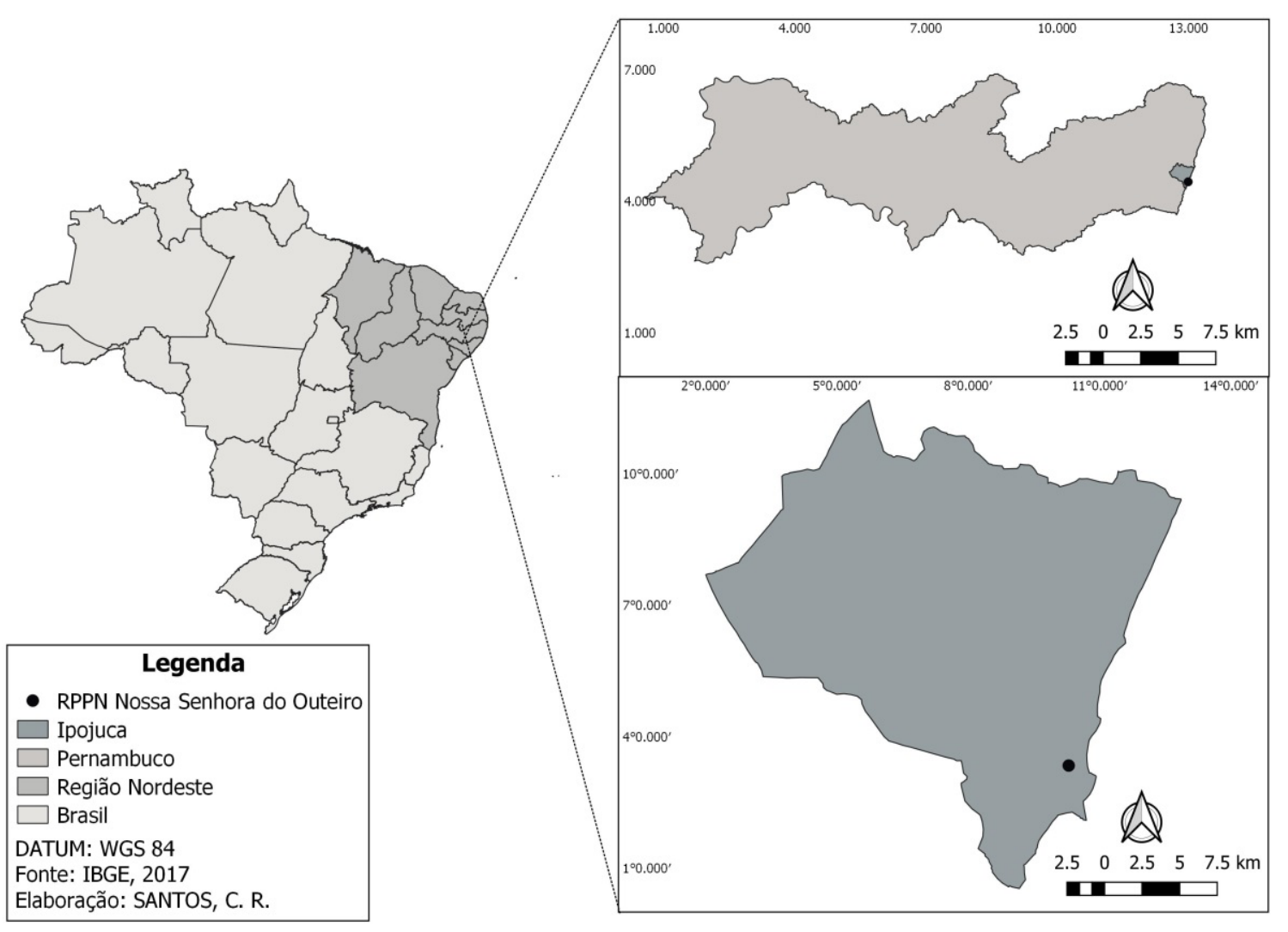

Figura 1. Mapa do Litoral pernambucano evidenciando a localização da RPPN Nossa Senhora do Outeiro de Maracaípe, Ipojuca, Pernambuco. Fonte: Santos, R.C. 
A restinga de Maracaípe foi selecionada para a avaliação da estrutura herbácea por ser considerada um dos poucos remanescentes de restinga bem conservados no Estado, de acordo com Almeida Jr. et al. (2009). Diferentes tipos fisionômicos foram descritos para a área (Almeida Jr. et al. 2009), sendo escolhido, para o presente estudo, a fisionomia campo não inundável.

\section{Coleta e amostragem do estrato herbáceo}

Para a amostragem da comunidade herbácea foi utilizado o método de parcelas proposto por Müeller-Dombois e Ellenberg (1974). Na área de estudo foram traçados cinco transectos (100 m cada) paralelos, com $10 \mathrm{~m}$ de distância entre si. As parcelas $\left(\right.$ de $1 \mathrm{~m}^{2}$ ) foram distribuídas a cada $10 \mathrm{~m}$ em cada transecção, alternadas em relação a linha do transecto, totalizando 50 parcelas.

Foram consideradas como estrato herbáceo as plantas não lenhosas e com caule verde (Araújo et al. 2005; Andrade et al. 2009), que possuíam altura entre 0,15e $1 \mathrm{~m}$. O limite mínimo de altura foi incluído para evitar possíveis erros de identificação das espécies. Os indivíduos jovens de espécies arbustivas ou arbóreas não foram considerados para essa amostragem. Cada planta teve o comprimento do maior eixo aéreo medido com auxílio de uma régua e o diâmetro do caule/pseudocaule ao nível do solo foi mensurado com auxílio de paquímetro digital. Cada planta foi considerada como único indivíduo desde que não apresentasse conexão com outra; no caso de indivíduos com ramos aéreos múltiplos (perfilhos), não individualizados ao nível do solo, foi contabilizado cada perfilho separadamente.

As plantas amostradas foram coletadas para posterior identificação, e a coleta do material seguiu a metodologia usual em botânica (Peixoto e Maia 2013). As espécies foram identificadas a partir de bibliografia especializada, chaves de identificação e por comparação do material depositado nos herbários de Pernambuco. Os exemplares que apresentaram dificuldade quanto à identificação ou que necessitavam de confirmação foram enviados a especialistas.

As espécies foram listadas seguindo a classificação do APG IV (2016) e a verificação da grafia das espécies bem como dos autores foi realizada através dos sites Tropicos (http://www.tropicos.org/) e Flora do Brasil 2020 (http://floradobrasil.jbrj. gov.br/). Nesse último, também foi consultada a origem e o padrão de distribuição das espécies nos domínios fitogeográficos. As exsicatas originadas do presente estudo foram incorporadas ao acervo do Herbário Vasconcelos Sobrinho (PEUFR), da Universidade Federal Rural de Pernambuco e no Herbário Dárdano de Andrade Lima (IPA), do Instituto de Pesquisa Agropecuária.

Para a análise da estrutura horizontal foram considerados os seguintes parâmetros fitossociológicos: densidade (absoluta e relativa), frequência (absoluta e relativa), dominância (absoluta e relativa), Índice de Diversidade de Shannon ( $\left.\mathrm{H}^{\prime}\right)$ e Índice de Equabilidade de Pielou (J'), utilizando o pacote FITOPAC 2.1 (Shepherd 
2006), além disso foram calculados o Valor de Importância (VI) e o Valor de Cobertura (VC). Os valores concernentes à densidade, frequência e dominância foram utilizados para determinar o VI, e a partir da soma da densidade relativa e dominância relativa foi obtido o VC. Para a caracterização da comunidade amostrada foram elaborados histogramas do número de indivíduos por intervalos de altura (amplitude de $10 \mathrm{~cm}$ ).

\section{RESULTADOS}

Foram amostradas 29 espécies (sendo duas morfoespécies), 22 gêneros e nove famílias (Tabela 1), sendo inventariado um total de 1.062 indivíduos. As famílias com maior riqueza específica foram Poaceae (com oito espécies), Cyperaceae (seis), Fabaceae, Euphorbiaceae e Rubiaceae (3 spp. cada). Juntas, essas famílias acumularam aproximadamente $79 \%$ do total de espécies amostradas. A maioria dos gêneros amostrados (86\%) apresentou uma única espécie, sugerindo alta diversidade genérica.

As espécies de maior VI foram Paspalum maritimum, Cuphea flava, Eleocharis geniculata, Stigmaphyllon paralias, Rhynchospora barbata, Stylosanthes viscosa, Eragrostis rufescens e Rhynchospora riparia. As espécies de maior frequência foram Paspalum maritimum (16,33\%), Stylosanthes viscosa (11,56\%) e Cuphea flava $(10,88 \%)$.

Quanto ao VC, as espécies foram as mesmas observadas no VI, havendo apenas alteração na ordem de algumas espécies, em que se destacaram Paspalum maritimum, Eleocharis geniculata, Cuphea flava, Rhynchospora barbata, Stigmaphyllon paralias e Eragrostis rufescens (Tabela 1). Dentre essas espécies, Cuphea flava, Stigmaphyllon paralias e Eragrostis rufescens são citadas como plantas nativas endêmicas do Brasil. Cabe destacar ainda que a espécie Cuphea flava é típica da Mata Atlântica ocorrendo de forma natural exclusivamente em ambientes de restinga.

Foram amostradas as espécies Paspalum arundinaceum Poir., típica de ambientes amazônicos e Croton sellowii Baill. que é característica de ecossistemas transicionais entre a Caatinga ea Mata Atlântica. A maioria das espécies inventariadas no presente estudo possui ampla distribuição nos domínios fitogeográficos brasileiros e, dentre essas, foram amostradas três espécies características de vegetação com indícios de antropização: Andropogon bicornis L., Euphorbia thymifolia L. e Schwenckia americana D. Royen ex L.

A altura média dos indivíduos amostrados foi de $38 \mathrm{~cm}$ formando um estrato herbáceo menos denso no campo. A altura mínima foi $15 \mathrm{~cm}$ sendo representada por seis espécies referentes às famílias Commelinaceae, Cyperaceae e Poaceae, e a altura máxima foi de $1 \mathrm{~m}$ representada por Stigmaphyllon paralias (Malpighiaceae). A 
distribuição do número de indivíduos por classes de altura evidenciou que cerca de $71 \%$ dos registros apresentaram até $45 \mathrm{~cm}$ (Figura 2).

O Índice de diversidade de Shannon (H') foi de 2,414 e o Índice de equabilidade de Pielou (J') encontrado foi de o,717.

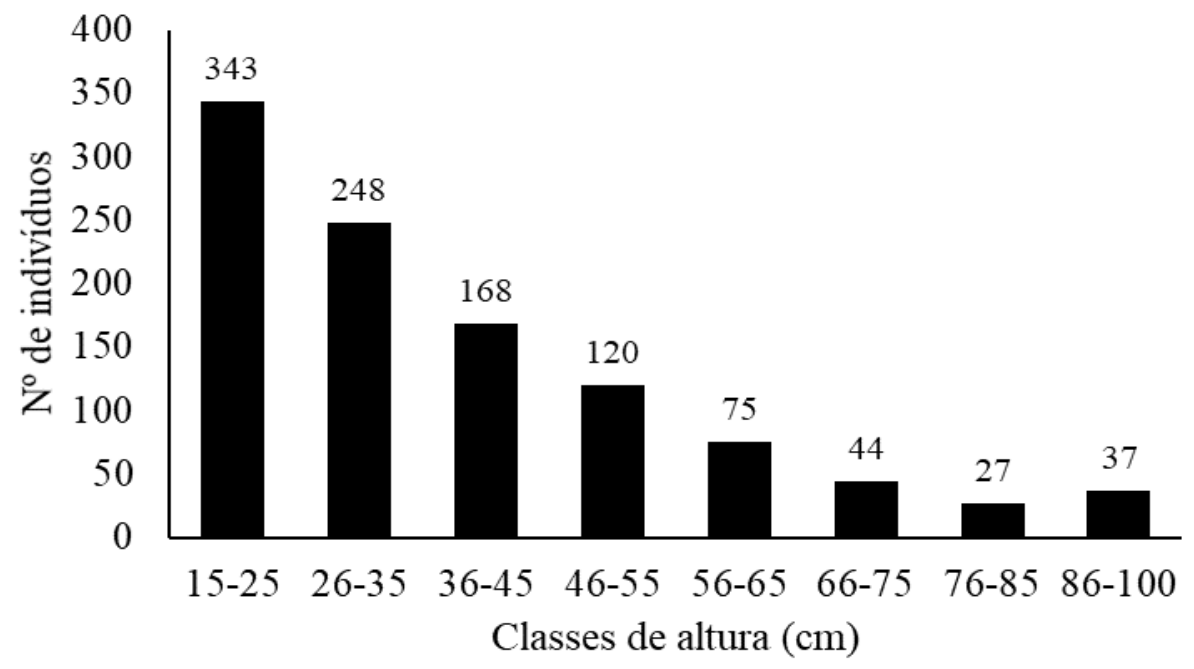

Figura 2. Distribuição, em classes de altura (em intervalos de $10 \mathrm{~cm}$ ), das espécies herbáceas amostradas no campo não inundável da restinga da Reserva Particular do Patrimônio Natural de Nossa Senhora do Outeiro de Maracaípe, Ipojuca, Pernambuco. 
Tabela 1. Fitossociologia do estrato herbáceo da fisionomia campo não inundável da Restinga de Maracaípe, Ipojuca, Pernambuco. NI = Número de Indivíduos; D.A. = Densidade Absoluta; D.R. = Densidade Relativa; F.A. = Frequência Absoluta; F.R. = Frequência Relativa; D.A. = Dominância Absoluta; D.R. = Dominância

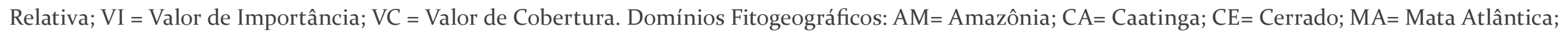

$$
\mathrm{PA}=\text { Pampas; } \mathrm{PN}=\text { Pantanal. }
$$

\begin{tabular}{|c|c|c|c|c|c|c|c|c|c|c|c|c|c|c|c|c|}
\hline \multirow{2}{*}{ Espécies } & \multirow{2}{*}{ Famílias } & \multicolumn{6}{|c|}{ Domínios Fitogeográficos } & \multirow{2}{*}{ N.I } & \multirow{2}{*}{ D.A. } & \multirow{2}{*}{ D.R. } & \multirow{2}{*}{ F.A. } & \multirow{2}{*}{ F.R. } & \multirow{2}{*}{ D.A. } & \multirow{2}{*}{ D.R. } & \multirow{2}{*}{ VI } & \multirow{2}{*}{ VC } \\
\hline & & AM & CA & CE & MA & PA & PN & & & & & & & & & \\
\hline Paspalum maritimum Trin. & Poaceae & $\mathrm{X}$ & $\mathrm{X}$ & $\mathrm{X}$ & $\mathrm{X}$ & - & - & 257 & 51400 & 24,18 & 48 & 16,33 & 1,71 & 10,57 & 51,07 & 34,74 \\
\hline Cuphea flava Spreng. & Lythraceae & - & - & - & $\mathrm{X}$ & - & - & 79 & 15800 & 7,43 & 32 & 10,88 & 3,81 & 23,53 & 41,84 & 30,96 \\
\hline $\begin{array}{l}\text { Eleocharis geniculata (L.) Roem. \& } \\
\text { Schult. }\end{array}$ & Cyperaceae & $\mathrm{X}$ & $\mathrm{X}$ & $\mathrm{X}$ & $\mathrm{X}$ & $\mathrm{X}$ & $\mathrm{X}$ & 89 & 17800 & 8,37 & 2 & 0,68 & 3,85 & 23,82 & 32,87 & 32,19 \\
\hline Stigmaphyllon paralias A.Juss. & Malpighiaceae & - & $\mathrm{X}$ & $\mathrm{X}$ & $\mathrm{X}$ & - & - & 184 & 36800 & 17,31 & 26 & 8,84 & 0,3 & 1,85 & 28 & 19,16 \\
\hline Rhynchospora barbata (Vahl) Kunth & Cyperaceae & $\mathrm{X}$ & $\mathrm{X}$ & $\mathrm{X}$ & - & - & - & 125 & 25000 & 11,76 & 14 & 4,76 & 1,26 & 7,81 & 24,33 & 19,57 \\
\hline Stylosanthes viscosa (L.) Sw. & Fabaceae & $\mathrm{X}$ & $\mathrm{X}$ & $\mathrm{X}$ & $\mathrm{X}$ & - & - & 77 & 15400 & 7,24 & 34 & 11,56 & 0,28 & 1,73 & 20,54 & 8,97 \\
\hline Eragrostis rufescens Schrad. ex Schult. & Poaceae & $\mathrm{X}$ & $\mathrm{X}$ & $\mathrm{X}$ & $\mathrm{X}$ & - & - & 54 & 10800 & 5,08 & 8 & 2,72 & 1,55 & 9,57 & 17,38 & 14,65 \\
\hline Rhynchospora riparia (Nees) Boeckeler & Cyperaceae & $\mathrm{X}$ & $\mathrm{X}$ & $\mathrm{X}$ & $\mathrm{X}$ & $\mathrm{X}$ & $\mathrm{X}$ & 17 & 3400 & 1,6 & 8 & 2,72 & 1,74 & 10,78 & 15,1 & 12,38 \\
\hline Commelina obliqua Vahl & Commelinaceae & $\mathrm{X}$ & $\mathrm{X}$ & $\mathrm{X}$ & $\mathrm{X}$ & - & - & 34 & 6800 & 3,2 & 10 & 3,4 & 0,28 & 1,71 & 8,31 & 4,91 \\
\hline Bulbostylis capillaris (L.) C.B.Clarke & Cyperaceae & $\mathrm{X}$ & $\mathrm{X}$ & $\mathrm{X}$ & $\mathrm{X}$ & $\mathrm{X}$ & $\mathrm{X}$ & 27 & 5400 & 2,54 & 10 & 3,4 & 0,13 & 0,78 & 6,72 & 3,32 \\
\hline Chamaecrista flexuosa (L.) Greene & Fabaceae & $\mathrm{X}$ & $\mathrm{X}$ & $\mathrm{X}$ & $\mathrm{X}$ & $\mathrm{X}$ & $\mathrm{X}$ & 10 & 2000 & 0,94 & 16 & 5,44 & 0,01 & 0,05 & 6,43 & 0,99 \\
\hline Steinchisma laxum (Sw.) Zuloaga & Poaceae & $\mathrm{X}$ & $\mathrm{X}$ & $\mathrm{X}$ & $\mathrm{X}$ & - & - & 21 & 4200 & 1,98 & 8 & 2,72 & 0,24 & 1,46 & 6,16 & 3,44 \\
\hline
\end{tabular}




\begin{tabular}{|c|c|c|c|c|c|c|c|c|c|c|c|c|c|c|c|c|}
\hline \multirow{2}{*}{ Espécies } & \multirow{2}{*}{ Famílias } & \multicolumn{6}{|c|}{ Domínios Fitogeográficos } & \multirow{2}{*}{ N.I } & \multirow{2}{*}{ D.A. } & \multirow{2}{*}{ D.R. } & \multirow{2}{*}{ F.A. } & \multirow{2}{*}{ F.R. } & \multirow{2}{*}{ D.A. } & \multirow{2}{*}{ D.R. } & \multirow{2}{*}{ VI } & \multirow{2}{*}{ VC } \\
\hline & & AM & CA & CE & MA & PA & PN & & & & & & & & & \\
\hline Cyperus meyenianus Kunth & Cyperaceae & $\mathrm{X}$ & $\mathrm{X}$ & $\mathrm{X}$ & $\mathrm{X}$ & $\mathrm{X}$ & $\mathrm{X}$ & 1 & 200 & 0,09 & 2 & 0,68 & 0,57 & 3,5 & 4,27 & 3,59 \\
\hline $\begin{array}{l}\text { Mitracarpus frigidus (Willd. ex Roem. \& } \\
\text { Schult.) K.Schum. }\end{array}$ & Rubiaceae & $\mathrm{X}$ & $\mathrm{X}$ & $\mathrm{X}$ & $\mathrm{X}$ & - & - & 9 & 1800 & 0,85 & 8 & 2,72 & 0,02 & 0,11 & 3,68 & 0,96 \\
\hline Poaceae 2 & Poaceae & - & - & - & - & - & - & 14 & 2800 & 1,32 & 6 & 2,04 & 0,05 & 0,3 & 3,66 & 1,61 \\
\hline Schwenckia americana Rooyen ex L. & Solanaceae & $\mathrm{X}$ & $\mathrm{X}$ & $\mathrm{X}$ & $\mathrm{X}$ & - & - & 4 & 800 & 0,38 & 6 & 2,04 & o & o & 2,42 & 0,38 \\
\hline Centrosema brasilianum (L.) Benth. & Fabaceae & $\mathrm{X}$ & $\mathrm{X}$ & $\mathrm{X}$ & $\mathrm{X}$ & $\mathrm{X}$ & - & 4 & 800 & 0,38 & 4 & 1,36 & 0,01 & 0,03 & 1,77 & 0,41 \\
\hline Euphorbia thymifolia L. & Euphorbiaceae & $\mathrm{X}$ & $\mathrm{X}$ & $\mathrm{X}$ & $\mathrm{X}$ & $\mathrm{X}$ & $\mathrm{X}$ & 2 & 400 & 0,19 & 4 & 1,36 & 0,03 & 0,17 & 1,71 & 0,35 \\
\hline Andropogon bicornis L. & Poaceae & $\mathrm{X}$ & $\mathrm{X}$ & $\mathrm{X}$ & $\mathrm{X}$ & $\mathrm{X}$ & $\mathrm{X}$ & 4 & 800 & 0,38 & 2 & 0,68 & 0,07 & 0,46 & 1,52 & 0,84 \\
\hline Rugoloa pilosa (Sw.) Zuloaga & Poaceae & $\mathrm{X}$ & - & - & $\mathrm{X}$ & - & - & 4 & 800 & 0,38 & 2 & 0,68 & 0,07 & 0,42 & 1,48 & 0,8 \\
\hline Croton sellowii Baill. & Euphorbiaceae & - & $\mathrm{X}$ & - & $\mathrm{X}$ & - & - & 4 & 800 & 0,38 & 2 & 0,68 & 0,02 & 0,11 & 1,17 & 0,49 \\
\hline Cyperus polystachyos Rottb. & Cyperaceae & $\mathrm{X}$ & $\mathrm{X}$ & $\mathrm{X}$ & $\mathrm{X}$ & $\mathrm{X}$ & $\mathrm{X}$ & 2 & 400 & 0,19 & 2 & 0,68 & o & o & 0,87 & 0,19 \\
\hline Poaceae 3 & Poaceae & - & - & - & - & - & - & 1 & 200 & 0,09 & 2 & 0,68 & 0,01 & 0,05 & 0,82 & 0,14 \\
\hline Paspalum arundinaceum Poir. & Poaceae & $X$ & - & - & - & - & - & 1 & 200 & 0,09 & 2 & 0,68 & $\mathrm{o}$ & o,o1 & 0,79 & 0,11 \\
\hline Morfoespécie 3 & Indeterminada & - & - & - & - & - & - & 1 & 200 & 0,09 & 2 & 0,68 & $\mathrm{o}$ & 0,01 & 0,78 & 0,1 \\
\hline Morfoespécie 2 & Indeterminada & - & - & - & - & - & - & 1 & 200 & 0,09 & 2 & 0,68 & o & o & 0,78 & 0,1 \\
\hline $\begin{array}{l}\text { Staelia virgata (Link ex Roem. \& Schult.) } \\
\text { K.Schum. }\end{array}$ & Rubiaceae & $\mathrm{X}$ & $\mathrm{X}$ & $\mathrm{X}$ & $\mathrm{X}$ & $\mathrm{X}$ & - & 1 & 200 & 0,09 & 2 & 0,68 & $\mathrm{o}$ & $\mathrm{o}$ & 0,78 & 0,1 \\
\hline Microstachys corniculata (Vahl) Griseb. & Euphorbiaceae & $\mathrm{X}$ & $\mathrm{X}$ & $\mathrm{X}$ & $\mathrm{X}$ & - & - & 1 & 200 & 0,09 & 2 & 0,68 & o & o & 0,78 & 0,1 \\
\hline
\end{tabular}




\section{DISCUSSÃO}

O levantamento fitossociológico do estrato herbáceo da restinga de Maracaípe evidenciou notória riqueza específica (29 espécies) quando comparado aos estudos fitossociológicos desenvolvidos nas restingas da Bahia por Dias e Menezes (2007) que registraram 11 espécies e Menezes et al. (2012) que contabilizaram 18 espécies para uma área de restinga ao sul e 20 espécies em uma restinga ao norte. Quando comparado aos estudos realizados no Maranhão, o presente estudo mostrou baixa riqueza, onde Amorim et al. (2016) registraram 58 espécies, Araujo et al. (2016) catalogaram 35 e Santos et al. (2019) amostraram 41 espécies.

Os valores observados nos estudos desenvolvidos na Bahia podem ser interpretados como baixos, devido à alta frequência, densidade e/ou dominância das espécies Cyperus pedunculatus (R.Br.) J. Kern, Euphorbia hyssopifolia, Marsypianthes chamaedrys (Vahl) Kuntze e Panicum racemosum (P. Beauv.) Spreng que contribuíram para o não desenvolvimento de outras espécies. Em relação aos estudos realizados no Maranhão, a riqueza pode estar associada a maior quantidade de espécies ruderais (Amorim et al. 2016), a presença de rizomas em muitas espécies (Araujo et al. 2016) ou à presença de plantas estoloníferas (Santos et al. 2019), conferindo as espécies uma certa vantagem na ocupação das áreas, afetando diretamente na riqueza da restinga.

Além disso, pode-se associar a riqueza da fisionomia campo a presença de espécies, principalmente, das famílias Poaceae, Cyperaceae que possuem adaptações a diversos tipos de ambientes, ocorrendo inclusive em áreas antropizadas, apresentarem mecanismos facilitadores de polinização e dispersão, o que pode favorecer o maior sucesso reprodutivo, contribuindo para a dominância dessas espécies (Palma e Jarenkow 2008; Sánchez-Ken e Clark 2010; Maciel e Alves 2011).

A abundância de Poaceae, Cyperaceae, Euphorbiaceae e Fabaceae foi relatada também por Menezes et al. (2012), Amorim et al. (2016) e Santos et al. (2019) quanto aos parâmetros fitossociológicos, o que pode estar relacionado a capacidade de se desenvolverem em ambientes de solos pobres como as restingas.

$\mathrm{O}$ índice de diversidade do presente estudo $\left(\mathrm{H}^{\prime}=2,414\right)$, apresentou menor valor diante dos estudos desenvolvidos no litoral do sul e sudeste do país, por Müller e Waechter (2001) com H' = 2,514; Klein et al. (2007) com H' = 3,25; Carvalho e Sá (2011) com H'= 2,90. Apresentou-se próximo ao encontrado por Amorim et al. (2016), com $H^{\prime}=2,47$, nas dunas de Araçagi, Maranhão; e inferior quando comparado aos valores registrados por Araújo et al. (2016), que obteve H'f = 3;04 nats.m² e Santos et al. (2019), com H’f= 3,12 nats.ind ${ }^{-2}$, que utilizaram a frequência para o cálculo dos índices. Esta diferença pode estar diretamente relacionada às distintas condições de conservação ou antropização das áreas. Por exemplo, algumas áreas são afastadas de moradias ou são em APA’s ou Reservas (como o presente estudo, Klein et al. 2007) e outras áreas apresentavam-se próximas a locais muito movimentados por banhistas ou pedestres, sendo observada degradação da vegetação devido à forte interferência humana. 
As espécies com maiores valores de frequência, Paspalum maritimum, Cuphea flava, Stylosanthes viscosa e Stigmaphyllon paralias também apresentaram maiores VI e VC; resultado também encontrado nas análises de herbáceas de uma restinga do Rio Grande do Sul, por Palma e Jarenkow (2008) e no Maranhão por Araújo et al. (2016). Felfili e Rezende (2003) destacaram que as espécies consideradas mais importantes em termos de VI são aquelas que apresentam o maior sucesso em explorar os recursos do seu habitat, sendo o VI um dado importante para caracterização e análise das relações intraespécies.

Quanto à densidade de indivíduos herbáceos um padrão já foi comprovado na literatura em que poucas espécies apresentam grande quantidade de indivíduos (Palma e Jarenkow 2008; Scarano 2009). Diante disso, a presença de espécies estoloníferas e rizomatosas pode, muitas vezes, influenciar na coleta de dados, superestimando a densidade dessas espécies devido ao elevado grau de cobertura (Almeida Jr. et al. 2009). Assim, Paspalum maritimum e Stigmaphyllon paralias se destacaram neste estudo por serem espécies rizomatosas e de crescimento clonal, respectivamente (Pereira et al. 2004; Oliveira et al. 2013), formando agrupamentos com uma eficiente propagação vegetativa por serem capazes de reter nutrientes e água, possibilitando sua sobrevivência em ambientes secos; além de maior resistência ao pisoteio, desmatamento e seca. Segundo Palma e Jarenkow (2008), o crescimento clonal torna-se vantajoso para muitas espécies que crescem em ambientes costeiros, sendo raro o estabelecimento por sementes. Em contrapartida, esse tipo de reprodução pode impedir que outras espécies possam se estabelecer devido à maior ocupação do solo como acontece com a P. maritimum que é tida como uma planta daninha na região Nordeste, infestando lavouras e ambientes antropizados, propagando-se com facilidade (Lorenzi 2008).

A maior contribuição da espécie $P$. maritimum deve-se à presença de rizomas e a sua capacidade de resistir ao pisoteio, fogo e seca (Maciel et al. 2009). Eleocharis geniculata também se destacou por possuir grande tolerância aos ambientes com maiores teores de salinidade (Gil e Bove 2007). E S. paralias apresentou elevado VI devido ao crescimento clonal e desenvolvimento em moitas (Pereira et al. 2004), preferencialmente em áreas abertas (Almeida Jr. et al. 2009), e em ambientes com altas temperaturas.

As espécies Croton sellowii, Cuphea flava, Eragrostis rufescens e Stigmaphyllon paralias são endêmicas do Brasil e juntas corresponderam a cerca de 30\% dos indivíduos amostrados no estudo. Cuphea flava que é típica da Mata Atlântica também ocorre em vegetação de restinga, evidenciando assim que as espécies que compõem a vegetação da área estudada são nativas e naturais de ambientes litorâneos. Dados diferentes foram encontrados por Araújo et al. (2016) que relataram a ausência de espécies endêmicas e atribuíram esse fato à formação geológica recente das restingas, confirmando a ocupação das áreas litorâneas por espécies provenientes de ecossistemas adjacentes (Rizzini 1979; Scarano 2002).

Croton sellowii e Stigmaphyllon paralias ocorrem em ambientes transicionais com a Mata Atlântica, como a Caatinga e o Cerrado, e podem habitar galerias que 
funcionam como corredores ecológicos para a colonização de outros biomas (Assis et al. 2004). Tais informações podem ter relação ainda com a influência na composição da vegetação das restingas pelos biomas circunvizinhos (Cerqueira 2000). Esses dados são importantes para conservação do fragmento estudado e para manutenção da diversidade da flora local. Tendo em vista que a composição vegetal é formada por espécies nativas e que compõem diferentes tipos vegetacionais, sendo de grande importância para a região nordeste como a Mata Atlântica, Caatinga e Cerrado (Assis et al. 2004; Giulietti et al. 2004).

Foram encontradas espécies que tem registros para os seis biomas brasileiros, como Andropogon bicornis, Bulbostylis capillaris, Chamaecrista flexuosa, Cyperus meyenianus, Cyperus polystachyos, Eleocharis geniculata, Euphorbia thymifolia e Rhynchospora riparia, dentre essas Andropogon bicornis, Euphorbia thymifolia, além da espécie Schwenckia americana, são características de ambientes antropizados. Esse comportamento generalista da vegetação que compõe a flora do litoral brasileiro é comum por conta das adaptações das espécies às diversas influências (Assis et al. 2004; Silva et al. 2008); a maior incidência de luz que se torna determinante para o desenvolvimento dos indivíduos herbáceos, os baixos teores de nutrientes dos solos (Santos-Filho et al. 2013), alta salinidade e ventos fortes (Machado e Almeida Jr. 2019) características comuns nas restingas do litoral nordestino.

\section{CONCLUSÃO}

Pode-se concluir que as espécies $P$. maritimum, $R$. barbata e $S$. paralias destacaram-se no arranjo estrutural da fisionomia campo; e a forma de crescimento e reprodução dessas espécies influenciaram diretamente na cobertura e entrada de diferentes espécies na área. Diante disso, deve-se ampliar os estudos ecológicos nos ecossistemas litorâneos para que se possa inferir sobre quais fatores apresentam maior peso quanto à ocorrência das espécies herbáceas em áreas de restinga, podendo apontar possíveis afinidades entre espécies ou grupos de espécies, auxiliando na compreensão da distribuição dessas espécies ao longo do litoral brasileiro.

\section{AGRADECIMENTOS}

Ao CNPq, pela concessão das bolsas de estudo do primeiro autor e de produtividade do último autor (proc. 301147/2004-3) e pelo financiamento do projeto "Aspectos Florísticos, Anatômicos e Ecológicos da vegetação da Restinga da RPPN Nossa Senhora do Outeiro de Maracaípe, Ipojuca, Pernambuco" (proc. 473974/o3-2), coordenado pela Profa. Dra. Carmen Silvia Zickel; aos especialistas de várias famílias que confirmaram e identificaram grande parte do material. 


\section{REFERENCIAS}

Almeida Jr. EB, Olivo MA, Araújo EL, Zickel CS. 2009. Caracterização da vegetação de restinga da RPPN de Maracaípe, Pernambuco, com base na fisionomia, flora, nutrientes do solo e lençol freático. Acta Botanica Brasilica, 23(1):36-48.

Almeida Jr. EB, Zickel CS. 2009. Fisionomia psamófila-reptante: riqueza de espécies na praia da pipa, Rio Grande do Norte, Brasil. Pesquisas Botânica, 6o:289-299.

Amorim IFF, Santos-Filho FS, Almeida Jr. EB. 2016. Fitossociologia do estrato herbáceo de uma área de dunas em Araçagi, Maranhão. In Almeida Jr. EB, Santos Filho FS. Biodiversidade do Meio Norte do Brasil: conhecimentos ecológicos e aplicações. Curitiba: CRV.

Andrade MVM, Andrade AP, Silva DS, Bruno RLA, Guedes DS. 2009. Levantamento florístico e estrutura fitossociológica do estrato herbáceo e subarbustivo em áreas de caatinga no cariri paraibano. Revista Caatinga, 22(1):229-237.

APG IV. 2016. An update of the Angiosperm Phylogeny Group classification for the orders and families of flowering plants: APG IV. Botanical Journal of the Linnean Society, 181(1):1-20.

Araújo EL, Silva KA, Ferraz AMN, Sampaio EVSB, Silva SI. 2005. Diversidade de herbáceas em microhabitats rochoso, plano e ciliar em uma área de caatinga, Caruaru, PE, Brasil. Acta Botânica Brasílica, 19(2):285-294.

Araújo ACM, Silva ANF, Almeida Jr. EB. 2016. Caracterização estrutural e status de conservação do estrato herbáceo de dunas da Praia de São Marcos, Maranhão, Brasil. Acta Amazonica, 46(3):247-258. http://dx.doi.org/10.1590/1809-4392201504265.

Assis AM, Thomas LD, Pereira OJ. 2004. Florística de um trecho de floresta de restinga no município de Guarapari, Espírito Santo, Brasil. Acta Botanica Brasilica, 18(1):191201.

Cantarelli JRR, Almeida Jr. EB, Santos-Filho FS, Zickel CS. 2012. Tipos fitofisionômicos e florística da restinga da APA de Guadalupe, Pernambuco, Brasil. Insula, 41:95-117.

Carvalho DD, Sá CFC. 2011. Estrutura do estrato herbáceo de uma restinga arbustiva aberta na APA de Massambaba, Rio de Janeiro, Brasil. Rodriguésia, 62(2):367-378.

Cerqueira R. 200o. Biogeografia de Restingas. Ecologia de Restinga e lagoas costeiras. Rio de Janeiro. NUPEM/UFRJ. 12p. 
Dias FJK, Menezes CM. 2007. Fitossociologia da vegetação sobre um cordão-duna no Litoral Norte da Bahia, Mata de São João, Brasil. Revista Brasileira de Biociências, 5 (2):1171-1173.

Felfili JM, Rezende RP. 2003. Conceitos e métodos em fitossociologia. Brasília: Universidade de Brasília, Departamento de Engenharia Florestal.

Flora do Brasil 2020 em construção. Jardim Botânico do Rio de Janeiro. Disponível em: $<$ http://floradobrasil.jbrj.gov.br/ >. Acesso em: 15 Jan. 2020.

Fuhro D, Vargas D, Larocca J. 2005. Levantamento florístico das espécies herbáceas, arbustivas e lianas da floresta de encosta da Ponta do Cego, Reserva Biológica do Lami (RBL), Porto Alegre, Rio Grande do Sul, Brasil. Pesquisas Botânica, 56:239-256.

Gil ASB, Bove CP. 2007. Eleocharis R. Br. (Cyperaceae) no estado do Rio de Janeiro, Brasil. Biota Neotropica, 7(1):163-193.

Gilliam FS. 2007. The ecological significance of the herbaceous layer in Temperate forest ecosystems. Bioscience, 57(10):845-858.

Giulietti AM, Bocage Neta AL, Castro AAJF, Gamarrarojas CFL, Sampaio EVSB, Virgínio JF, Harley RM. 2004. Diagnóstico da vegetação nativa do bioma caatinga. Biodiversidade da Caatinga: áreas e ações prioritárias para a conservação.

INMET - Instituto Nacional de Meteorologia. [acess 2017 jan. 10]. Available from: http://www.inmet.gov.br.

Klein AS, Citadini-Zanette V, Santos R. 2007. Florística e estrutura comunitária de restinga herbácea no município de Araranguá, Santa Catarina. Biotemas, 20(3):15-26.

Köppen W. 1948. Climatologia: com un estudio de los climas de la tierra. $\mathbf{1}^{\mathrm{a}}$ ed. Mexico: Fondo de Cultura Economica.

Lorenzi H. 2008. Plantas daninhas do Brasil: terrestres, aquáticas, parasitas e tóxicas. $4^{\underline{a}}$ ed. São Paulo: Instituto Plantarum.

Machado MA, Almeida Jr. EB. 2019. Spatial structure, diversity, and edaphic factors of an area of Amazonian coast vegetation in Brazill. Journal of the Torrey Botanical Society, 146(1):58-68.

Maciel JR, Alves MA. 2011. Família Poaceae na Serra de Itabaiana, Parque Nacional Serra de Itabaiana, Sergipe-Brasil. Revista Caatinga, 24(3):85-93. 
Maciel JR, Oliveira JC, Alves M. 2009. Paspalum L. (Poaceae: Panicoideae: Paniceae) no estado de Pernambuco, Brasil. Acta Botanica Brasilia, 23(4):1145-1161.

Martins FR, Batalha MA. 2011. Formas de vida, espectro biológico de Raunkiaer e fisionomia da vegetação. Fitossociologia no Brasil: métodos e estudos de casos, 1:4485.

Menezes CM, Espinheira MJCL, Dias FJK, Silva VIS. 2012. Composição florística e fitossociologia de trechos da vegetação praial dos litorais norte e sul do estado da Bahia. Revista Biociência, 18(1):35-41.

Müeller-Dombois D, Ellenberg H. 1974. Aims and methods of vegetation ecology. New York: John Wiley.

Müller SC, Waechter JL. 2001. Estrutura sinusial dos componentes herbáceo e arbustivo de uma floresta costeira subtropical. Revista Brasileira de Botânica, 24(4):395-406.

Munhoz CBR, Felfili JM. 20o8. Fitossociologia do estrato herbáceo-subarbustivo em campo limpo úmido no Brasil Central. Acta Botanica Brasilica, 22(4):905-913.

Oliveira DG, Prata AP, Ferreira RA. 2013. Herbáceas da Caatinga: composição florística, fitossociologia e estratégias de sobrevivência em uma comunidade vegetal. Revista Brasileira de Ciências Agrárias, 8(4):623-633.

Palma CB, Jarenkow JA. 2008. Estrutura de uma formação herbácea de dunas frontais no litoral norte do Rio Grande do Sul, Brasil. Biociências, 16(2):114-124.

Peixoto AL, Maia LC. 2013. Manual de procedimentos para herbários: INCT Herbário virtual para a Flora e os Fungos. a $^{\mathrm{a}}$ ed. Recife: Editora Universitária UFPE.

Pereira MCA, Cordeiro SZ, Araújo DSD. 2004. Estrutura do estrato herbáceo na formação aberta de Clusia do Parque Nacional da Restinga de Jurubatiba, RJ, Brasil. Acta Botanica Brasilica, 18 (3):677-687.

Rizzini CT. 1979. Tratado de Fitogeografia do Brasil. HUCITEC EDUSP, São Paulo, 374p.

Sacramento AC, Zickel CS, Almeida Jr. EB. 2007. Aspectos florísticos da vegetação de restinga no litoral de Pernambuco. Revista Árvore, 31(6):1121-1130.

Sánchez-Ken JG, Clark LG. 2010. Phylogeny and a new tribal classification of the Panicoideae s.l. (Poaceae) based on plastid and nuclear sequence data and structural data. American Journal of Botany, 97:1732-1748. 
Santos RS, Amorim IFF, Almeida Jr. EB. 2019. Caracterização fitossociológica do componente halófilo-psamófilo em uma área de dunas, São Luís, Maranhão, Brasil. Boletim do Laboratório de Hidrobiologia, 29:1-8.

Santos-Filho FS, Almeida Jr. EB, Soares CJRS, Zickel CS. 2015. Flora and woody vegetation structure in an insular area of restinga in Brazil. International Journal of Ecology and Environmental Sciences, New Delhi, 4(3):157-16o.

Santos-Filho FS, Almeida Jr. EB, Zickel CS. 2013. Do edaphic aspects alter vegetation structures in the Brazilian restinga? Acta Botanica Brasilica, 27(3):613-623.

Scarano FR. 2002. Structure, function and floristic relantioships of plants communities in stressful habitats marginal to Brazilian Atlantic Rainforest. Annals of Botany, 90:517-524.

Scarano FR. 2009. Plant communities at the periphery of the Atlantic rain forest: Rarespecies bias and its risks for conservation. Biological Conservation, 142:1201-1208.

Shepherd GJ. 2006. Fitopac V. Campinas, Universidade Estadual de Campinas Departamento de Botânica.

Silva ANF, Araújo ACM, Almeida Jr. EB. 2016. Flora fanerogâmica das dunas da Praia de São Marcos, São Luís, Maranhão. In: Almeida Jr. EB, Santos-Filho FS. Biodiversidade do Meio Norte do Brasil: conhecimentos ecológicos e aplicações. Curitiba: CRV.

Silva SSL, Zickel CS, Cestaro LA. 2008. Flora vascular e perfil fisionômico de uma restinga no litoral sul de Pernambuco. Acta Botanica Brasilica, 22(4):1123-1135.

Solos Embrapa. 2013. Sistema brasileiro de classificação de solos. Rio de Janeiro: Centro Nacional de Pesquisa de Solos.

Sugiyama M. 1998. Estudo de floresta da restinga da Ilha do Cardoso, Cananéia, São Paulo, Brasil. Boletim do Instituto de Botânica, 11:119-159. 